This is the submitted version of the article:

González-Monje P., Ayala García A., Ruiz-Molina D., Roscini C.. Encapsulation and sedimentation of nanomaterials through complex coacervation. J ournal of Colloid and Interface

Science, (2021). 589. : 500 - . 10.1016/j.jcis.2020.12.067.

Available at: https://dx.doi.org/10.1016/j.jcis.2020.12.067 


\title{
Encapsulation and sedimentation of nanomaterials through complex coacervation
}

\author{
Pablo González-Monje, Alex Ayala García, Daniel Ruiz-Molina, Claudio Roscini* \\ ICN2 (CSIC \& BIST), Campus UAB, Bellaterra, 08193 Barcelona, Spain \\ *Corresponding author. \\ E-mail addresses: dani.ruiz@icn2.cat (D. Ruiz-Molina); claudio.roscini@icn2.cat (C. Roscini)
}

\begin{abstract}
Hypothesis: Nanoparticles from seawage water is a health and environmental challenge, due to the increasing use of these materials of excellent colloidal stability. Herein we hypothesize to reach this objective through complex coacervation, a straightforward, low-cost process, normally accomplished with non-toxic and biodegradable macromolecules. Highly dense polymer-rich colloidal droplets (the coacervates) obtained from a reversible charge-driven phase separation, entrap suspended nanomaterials, allowing their settling and potential recovery.
\end{abstract}

Experiments: In this work we apply this process to highly stable aqueous colloidal dispersions of different surface charge, size, type and state (solid or liquid). We systematically investigate the effects of the biopolymers excess and the nanomaterials concentration and charge on the encapsulation and sedimentation efficiency and rate. This strategy is also applied to real laboratory water-based wastes.

Findings: Long-lasting colloidal suspensions are succesfully destabilized through coacervate formation, which ensures high nanomaterials encapsulation efficiencies ( 85\%), payloads and highly transparent supernatants (\%T $~ 90 \%)$, within two hours. Lower polymer excess induces faster clearance and less sediments, while preserving effective nanomaterials removal. Preliminary experiments also validate the method for the clearance of real water residuals, making complex coacervation a promising scalable, low-cost and ecofriendly alternative to concentrate, separate or recover suspended micro/nanomaterials from aqueous sludges.

KEYWORDS: Microencapsulation, Complex coacervation, Biopolymers, Nanoparticles recovery, Nanomaterials removal

Abbreviations: SEM, Scanning Electron Microscopy; OM, Optical Microscopy; DLS, Dy- namic Light Scattering; UV-Vis, Ultraviolet-Visible; 1H NMR, proton nuclear magnetic resonance; DR13, Disperse Red 13; G, Gelatin; GA, gum Arabic; (士)PS-NPs, Polystyrene Nanoparticles positively/negatively charged; Au-NPs, gold nanoparticles; SiOx-NPs, sili- con oxide nanoparticles; o/w-NE, oil-in-water nanoemulsion; Mow-NPs, Mowlith ${ }^{\circledR}$ LDM 7510 acrylate nanoparticles; Mixed-NPs, mixture of nanoparticles; Lab1-WE, Lab2-WE, laboratory aqueous wastes; nanomaterial@G-GA, capsules obtained from the entrapment of the nanomaterials; TSLs, solids and non-volatile liquids; EE, encapsulation efficiency. 


\section{Introduction}

Coacervation is the process in which a phase separation from a liquid medium is induced as result of the controlled modification of specific physico-chemical environmental conditions (e.g. pH, solubility, ionic strength, temperature, etc.), which yields two distinct liquid phases: the colloidal polymer-rich phase (the coacervate) and a diluted polmer phase (the supernatant) [1-3]. In complex coacervation, the associative electrostatic interactions of two oppositely charged polyelectrolytes initially soluble in water, undergo reciprocal and reversible complexation upon temperature and/or $\mathrm{pH}$ changes, yielding an insoluble hydrophilic macromolecules complex embedding a high amount of water (the coacervate) [4]. The coacervates phase appears as amorphous liquid droplets, which upon coalescence may separate out from the water by gravity. Complex coacervation is a relatively low-cost process occurring under non-extreme experimental conditions (e.g. avoiding toxic organic solvents) and using broadly available synthetic and/or natural, biodegradable, biocompatible and food-grade macro- molecules (e.g. polysaccharides and proteins) [5-6]. For these features, complex coacervation has been proposed and employed for a large variety of materials and applications: preparation of micelles and polymer micro/nanoparticles [1,4], purification of macromolecules [7], formation of biodegradable edible films [8], hydrogels [9], as water hardness indicator [10], and thermally-responsive underwater adhesives [11].

Nevertheless, given the achievable high payload and encapsulation efficiency ( $>95 \%)$, complex coacervation has been most broadly used as effective micro/nanoencapsulation method in pharmaceutical, agricultural and cosmetics [2], for the stabilization against oxidation or other degradation processes of food and agriculture ingredients [12], health-beneficial biofunctional oils or oil soluble compounds [13], for the controlled release of pesticides, insect repellents, fragrances [14] and drugs [15], and for the confinement of microstructured phase change materials used in energy storage [16], among others.

In the encapsulation process, in the presence of colloidal dispersions (e.g. oil-in-water emulsion), the coacervates distributes on the interface forming a uniform coating, which jellifies upon cooling, yielding capsules [2-3]. The control of the experimental conditions (e.g. $\mathrm{pH}$, type of polymer and core material, concentration, core/shell materials ra- tio, stirring rate, etc.), directly affects the morphology and structure of the coacervates which could form mononuclear or multinuclear capsules (containing one or more core compartments) [17-20], highly dispersed nanospheres, aggregated nanoparticles or a continuous polymer network [21].

Herein we propose the straightforward and scalable complex coacervation process as an alternative to well-established techniques aimed to entrap and separate suspended fine nanomaterials from aqueous sus- pensions, for their removal, recovery and recycling. Current separation methods of suspended particles are mostly based on coagulation and flocculation processes [22-25], induced by inorganic additives or polymer flocculants, which however rise health and ecotoxicity concerns [26-27], or require cost and time-consuming chemical syntheses or modifications (grafting) of polymeric flocculants [28-35], to increase the removal efficiency. Besides, coagulation and flocculation produce ir- reversible aggregations which prevent any possible recovery or recycling of the precipitated nanomaterials [26,31]. Our hypothesis is that under 
suitable conditions, the coacervate entraps the suspended nanomaterials forming a highly dense polymer network that upon gelation settles with the encapsulated material (Scheme 1) $[2,20]$. On top of that, the use of low-cost/non-toxic biodegradable natural polymers and the reversible $\mathrm{pH}$-controlled polymer charge formation, which could be of interest for the recovery of encapsulated high-added value nanomaterials (e.g. precious and catalytic nanoparticles), may represent a unique strategy for nanomaterials removal and/or recovery. Recently, coacervation has been employed as ecofriendly bioadsorbents to efficiently and reversibly adsorb/extract organic dyes from water homogeneous solutions [36-38], though as far as we know, a systematic study to validate its use to entrap and settle nanomaterials from aqueous sludge is lacking.

In this work gum Arabic (GA) and gelatin (G) were chosen as natural, readily available, biodegradable, low-toxic model amphoteric biopolymers, whose charge can be finely controlled by the $\mathrm{pH}$ [39]. We first investigated the feasibility of the coacervationinduced encapsulation and settling of model nanoparticles of different dimensions (10$700 \mathrm{~nm}$ ), phase (solid/liquid), surface charge, and nature (organic, inorganic) and evaluate the effect of changing the experimental para- meters. Finally, complex coacervation was applied to micro/nanoparticles-reach real water residues generated in our laboratories (Table S1), to explore the possibility of using this process as alternative strategy to sediment micro/nanomaterials from water wastes for their removal.

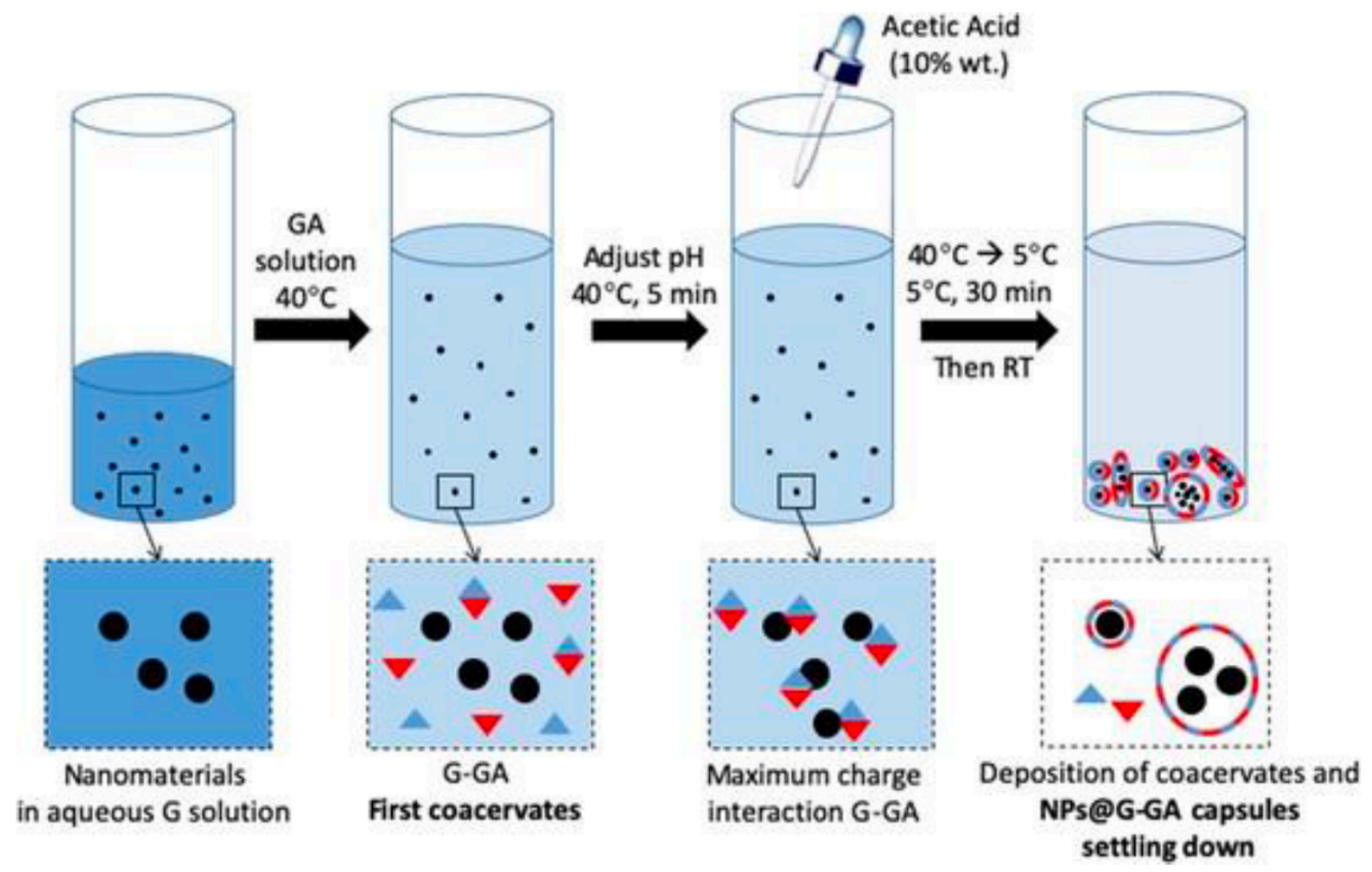

Scheme 1. Representation of the complex coacervation-induced nanomaterials encapsulation and settling. 


\section{Experimental section}

\subsection{Materials and chemicals}

Silicon oxide nanoparticles (SiOx-NPs), gold nanoparticles (Au-NPs), negatively and positively charged polystyrene nanoparticles ((-)PS-NPs, (+)PS-NPs) and oil (Miglyol ${ }^{\circledR}$ 812 ) in water nanoemulsion (o/w-NE) were synthesized in our laboratory and the procedures are described in the Supporting Information. ${ }^{\circledR}$ Mowilith LDM 7510 was acquired from UNIVAR ${ }^{\circledR}$ and used as received. A simulated aqueous effluent made of a mixture of the previous nanoparticles (Mixed-NPs) was also prepared with mass ratio (JPS-NPs:SiOx-NPs:Au-NPs:o/ w-NE = 1.43:1.43:1.00:2.71. The dye Disperse Red 13 (DR13) and polystyrene (PS) were acquired from Sigma Aldrich. The oil Miglyol ${ }^{\circledR} 812$ was acquired from Oxi-Med Express (distributor of Sasol in Spain). The surfactants sodium dodecyl sulphate (SDS) or hexadecyltrimethylammonium bromide (CTAB) were obtained from Sigma-Aldrich. Laboratory aqueous waste effluents (Lab1-WE and Lab2WE) were collected respectively from two different chemistry laboratories both containing a high concentration of micro/nanoparticles of different nature and size, emulsified oils and organic solvents, dyes, pigments, salts, reactants. Gelatin from porcine skin type $A$ and $~ 300 \mathrm{~g}$ bloom $(G)$ and gum Ara- bic from acacia tree (GA) were purchased and used as received from Sigma-Aldrich. Milli- $Q^{\circledR}$ water was used in all model experiments.

\subsection{Description of the complex coacervation process.}

The encapsulation and settling of nanomaterials present in aqueous streams by complex coacervation with G-GA systems was performed as showed in Scheme 1. G and GA mass ratio 1:1 was used for all experiments varying polymers ( $G+G A)$ :NPs mass ratio by changing the amount of polymers or NPs. Initially, both aqueous $\mathrm{G}$ and GA solutions were prepared with Milli- $Q^{\circledR}$ water at $40{ }^{\circ} \mathrm{C}$ and mild agitation $(250 \mathrm{rpm})$. Afterwards, NPs suspension or oil-in-water nanoemulsions or lab-wastes (concentrations specified in the text) were firstly poured into the gelatin solution $(10 \mathrm{ml})$ at $40^{\circ} \mathrm{C}$ and left under mild agitation $(250 \mathrm{rpm})$ for $5 \mathrm{~min}$. Next GA solution $(10 \mathrm{ml})$ was added and left at $40{ }^{\circ} \mathrm{C}$ and $250 \mathrm{rpm}$ for another $5 \mathrm{~min}$ (G:GA mass ratio $=1: 1$ ). In the experiments where $\mathrm{G}$ and GA amount was maintained constant, $G$ and $G A$ concentration was fixed at $10 \mathrm{mg} / \mathrm{ml}$. In the experiments where the polymers amount was varied, the $G$ and $G A$ solutions volume was maintained constant, while the $G$ and GA concentration was modified, accordingly (always maintaining G:GA mass ratio $=1: 1$ ). Successively the $\mathrm{pH}$ was adjusted at $4.0 \pm$ 0.1 by adding, drop-wise, a $10 \mathrm{wt} \%$ acetic acid solution. Once the $\mathrm{pH}$ was adjusted coacervates formed effectively because of maximum electrostatic interactions, yielding a hazy solution and indicating the coacervates formation.

The temperature was gradually decreased until it reached room temperature (RT), at which the microcapsules were already formed, and finally lowered to $5-10{ }^{\circ} \mathrm{C}$, keeping the temperature for $30 \mathrm{~min}$ under mild agitation conditions (200 rpm) to allow the gelation of gelatine. Magnetic stirring was then stopped and the formed large nanomaterials@G-GA microcapsules were let settling down. 


\subsection{Characterization techniques}

\subsubsection{Optical microscopy (OM)}

Digital images of nanomaterials@G-GA microcapsules were collected through the Zeiss Primo Star optical microscope and AxioCam camera. The nanomaterials@G-GA microcapsules water suspensions $(\sim 100 \mu \mathrm{l})$ were deposited on the glass substrate. Images were taken be- fore and after the water evaporation.

\subsubsection{Scanning electron microscopy (SEM)}

Images were collected through the scanning electron microscope FEI Quanta 650 FEG at acceleration voltages between 2 and $10 \mathrm{kV}$. Samples were mounted on SEM metal stubs covered with aluminum tape and were coated with a thin layer of platinum ( 5 $\mathrm{nm})$. Microcapsules sus- pensions $(\sim 100 \mu \mathrm{l})$ were deposited on the stub by drop casting, allowing solvent evaporation in air at room temperature.

\subsubsection{Transmission electron microscopy (TEM)}

Au-NPs images were collected from the transmission electron micro- scope Hitachi $\mathrm{H}-7000$. For the sample preparation, a diluted Au-NPs suspension ( $10-20 \mu \mathrm{l})$ was deposited onto a TEM grid.

\subsubsection{UV-Vis spectroscopy}

Turbidimetry was performed with Agilent Cary 60 UV-Vis spectrophotometer by measuring the variation of the optical transmittance of the suspension in $1 \mathrm{~cm}$ cuvette caused by the changes of the light scattered by the NPs. The absorption and transmission spectra of the suspensions were obtained in transmittance mode, without integrating sphere. Time-dependent absorption and transmission spectra were collected upon maintaining the cuvette standing (without stirring). The final supernatant fractions of the treated samples were measured by transferring the clear higher part of the decanted suspension, after keeping the container standing for $24 \mathrm{~h}$ without stirring. Sedimentation rate was monitored following the increase of \%T upon time at $400 \mathrm{~nm}$. For all samples the suspension clearing rate is obtained measuring the $\% \mathrm{~T}$ at $400 \mathrm{~nm}$ (\%T400tx) at $0 \mathrm{~min}, 5 \mathrm{~min}$ and $2 \mathrm{~h}$ after the coacervation is finished and stirring is stopped (the treated suspension is let settling with- out stirring). \%T40024h was instead measured for the supernatant solutions extracted from the standing suspension (kept without stirring) $24 \mathrm{~h}$ after the treatment.

\subsubsection{Thermogravimetric analysis (TGA)}

Payload and entrapment efficiency of silica nanoparticles (SiOx-NPs) was determined by mass loss in thermogravimetric analysis (TG). Thermogravimetric analysis of the SiOxNPs@G-GA microcapsules was carried out in with NETZSCH STA 449 F1 Jupiter using 2 $\mathrm{mg}$ of samples and heating with a rate of $10^{\circ} \mathrm{C} / \mathrm{min}$. 


\subsubsection{Proton nuclear magnetic resonance spectroscopy (1H-NMR)}

The payload (weight of encapsulated nanomaterial respect to the nanomaterials@capsules weight) and the corresponding encapsulation efficiency (EE, amount of encapsulated nanomaterials respect to the initial amount, before treatment) provided for polystyrene- (( \pm PS-NPs@G-GA) and oil-containing (o/w-NE@G-GA) capsules were determined by $1 \mathrm{H}$ NMR after breaking/swelling them by stirring/sonicating in $\mathrm{CDCl} 3$ for $24 \mathrm{~h}$ at $20-40{ }^{\circ} \mathrm{C}$. $20-35 \mathrm{mg}$ of microcapsules were dissolved in $\mathrm{CDCl} 3$ and $1 \mathrm{H}$ NMR spectra were recorded using the Bruker DPX250 (250 $\mathrm{MHz}$ ) spectrometer. The spectra are given in chemical shifts, $\delta(\mathrm{ppm})$. The peaks are defined as singlets $(\mathrm{s})$, triplets $(\mathrm{t})$ or multiplets $(\mathrm{m}) .17-20 \mathrm{mg}$ of DMF $(\delta=2.88(\mathrm{~s}, 3 \mathrm{H}$, $\mathrm{CH} 3), 2.97(\mathrm{~s}, 3 \mathrm{H}, \mathrm{CH} 3)$,

$7.96(\mathrm{~s}, 1 \mathrm{H}, \mathrm{CH}))$ were added to the samples, as an internal reference, for quantitative determination of the microcapsules payload (Miglyol ${ }^{\circledR} 812$ oil and PS).

\subsubsection{Dynamic light scattering (DLS) and z-potential}

The average size and their z-potential of the NPs suspensions were analyzed, after diluting, by dynamic light scattering (DLS) with the Malvern Zetasizer NanozS equipment and using a transparent plastic cuvette.

\subsubsection{Freeze-drying}

Approximately $10-20 \mathrm{ml}$ of the microcapsules suspension were frozen at $-83^{\circ} \mathrm{C}$ and then freeze-dried during two/three days in a $600 \mathrm{ml}$ flask and using the Telstar Cryodos 50 .

\subsubsection{Digital videos and photographs}

Digital videos and photographs of the suspensions were obtained using the photocamera of the Xiaomi Mi 5 s mobile phone.

\section{Results and discussion}

\subsection{Encapsulation and sedimentation of polystyrene nanoparticles}

The nanomaterials encapsulation and sedimentation through com- plex coacervation was first validated with negatively charged poly- styrene nanoparticles ((-)PS-NPs), integrating the red-colored Disperse Red 13 (DR13) dye, synthesized in our laboratory using a well-established nanoemulsion-phase separation method (the nanoparticles syn- thesis is detailed in the Supporting Information) [40-41]. The dye integration aimed to facilitate the tracking along the coacervation-induced entrapment and settlement processes of the (-)PS-NPs. The resulting red colored NPs displayed average diameter of $\sim 180 \mathrm{~nm}$, according to DLS measurements, in agreement with SEM images (Fig. 1a-b and S1) and a z-potential value of $-33.2 \mathrm{mV}$ provided by the sodium dodecyl sulfate (SDS) surfactant (Table S1). Time-dependent DLS measurements confirmed that the nanoparticles suspension $(0.0250 \mathrm{mg} / \mathrm{ml})$ was highly stable for more 
than a week (Table S2, Fig. 1b) with no sediments visually detected even after months of storage without stirring.

The encapsulation of the nanoparticles was carried out using a mixture of gelatin (G) and gum Arabic (GA) of 1:1 mass ratio, which guarantees the maximum electrostatic interaction [39,42-43]. The concentration of NPs was fixed to $0.0250 \mathrm{mg} / \mathrm{ml}$ while three different concentrations of the polymeric mixture $(0.1-2.5 \mathrm{mg} / \mathrm{ml})$ were used, which equates to mixture polymers $(G+G A)$ :NP mass ratios of 100:1, 40:1, and 4:1. The detailed procedure of the coacervation process is described in the Experimental Section. Visual inspection of the three experiments revealed that, once the coacervation was induced and the stirring was stopped, within a few minutes most of the (-)PS-NPs were settled at the bottom of the vial in the form of large coacervate particles, while clear supernatant solutions were obtained (see Figure $\mathbf{S} \mathbf{2}$ in Supporting In-formation).

a)
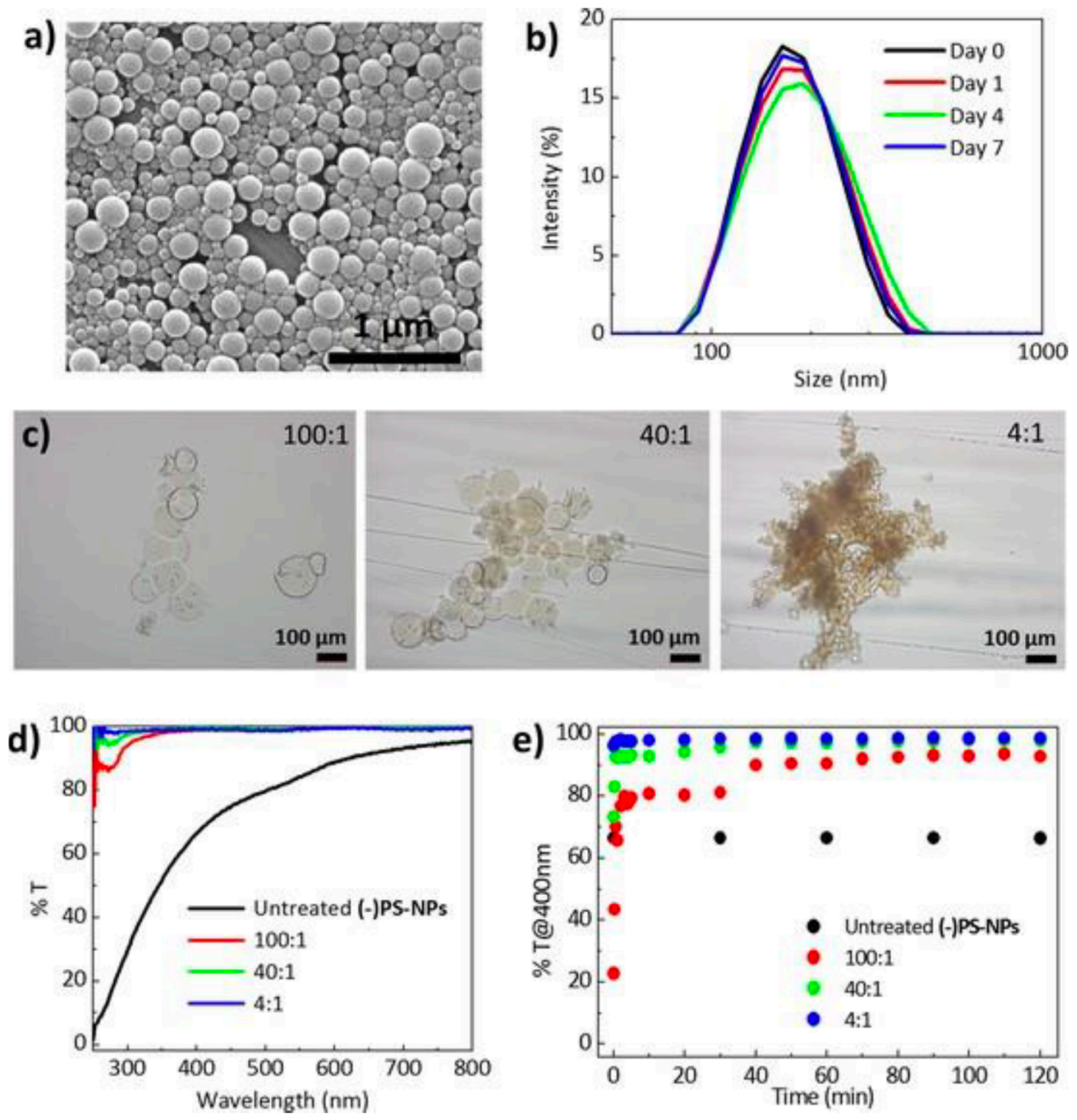

Fig. 1. a) SEM image of untreated (-)PS-NPs; b) DLS measurements of untreated (-)PS-NPs suspension $(0.0250 \mathrm{mg} / \mathrm{ml})$ during 7 days; c) OM images of (-)PS-NPs@G-GA microcapsules obtained using polymers:NPs mass ratios of 100:1, 40:1 and 4:1 and fixing the concentration of (-)PS-NPs $(0.5 \mathrm{mg}, 0.0250 \mathrm{mg} / \mathrm{ml}) ; d)$ transmittance spectra of (-)PS-NPs suspension $(0.0250 \mathrm{mg} / \mathrm{ml})$ before treatment and of the supernatant solutions obtained from the treated suspensions (using polymers:NPs mass ratios of 100:1, 40:1 and 4:1) after $24 \mathrm{~h}$ of standing; $e$ ) time-dependent \%T400 of the (-)PSNPs suspension $(0.0250 \mathrm{mg} / \mathrm{ml})$, untreated and after treatment using polymers:NPs mass ratios of 100:1, 40:1 and 4:1 and stopping the magnetic stirring. 
Optical (OM) and scanning electron microscopy (SEM) analysis of the sediment revealed the presence of microcapsules ((-)PS-NPs@G-GA) of different morphologies and dimensions, ranging from 50 to $100 \mu \mathrm{m}$, depending on the coacervation conditions used (see Fig. 1c and S3-S4).

Well-defined spherical shape (-)PS-NPs@G-GA microcapsules were found with polymers:NP mass ratios of 100:1 and 40:1, whereas irregular shape microstructures were obtained when the lowest polymers excess (i.e. 4:1 mass ratio) was used. A similar shape dependency on the wall/core weight ratio has been previously described for oilcore G-GA microcapsules, which at lower biopolymers excess tend to aggregate into irregular structures [17-20]. In our system we ascribed this effect to the presence of small amount of coacervates sur- rounding each NPs, destabilizing them and inducing their aggregation due to the high surface tension. Moreover, the higher concentration of NPs within the coacervates (in the case of using polymers:NP 4:1 mass ratio) might cause the decrease and widening of the gelation temperature of the complex [20]. Therefore, once the stirring is stopped, the still liquid-phase coacervates aggregate and coalesce forming a large highly dense polymer network containing the NPs.

The reddish color of the sedimented microcapsules observed at the optical microscope suggests the encapsulation of the DR13-containing (-)PS-NPs (Fig. 1c). The sedimentation process was quantitatively followed by UV-Vis spectroscopy (Fig. 1d-e and S5-S6 and Table 1), which for the initial untreated (-)PS-NPs suspension showed the typical transmittance curve of highly scattering particles, with the $\% \mathrm{~T}$ decreasing at shorter wavelengths (Fig. 1d). The little shoulder at $\lambda=530 \mathrm{~nm}$ was due to the absorption of the DR13 dye. Time-dependent optical measurements at $\lambda=400 \mathrm{~nm}$ (the visible wavelength providing the highest scattering), carried out by monitoring the variation of the sus- pension transparency \%T400tx at a given time tx (Fig. 1e and S6, Table 1), after completing the coacervation process and keeping the resulting suspension standing without stirring, provided information on the clearing rate and efficiency. The results revealed that the sedimentation rate depends on the polymers:NP mass ratio, i.e. the G-GA excess respect to the (-)PS-NPs. Immediately and $5 \mathrm{~min}$ after the gelation and the stirring was stopped, the mixtures treated with higher polymer amount, displayed the lowest transmittance values $\left(\% \mathrm{~T}^{400}{ }_{\text {to\&5min }}(100: 1)<\right.$ $\% \mathrm{~T}^{400}$ t0\&5min $\left.(40: 1)<\% \mathrm{~T}^{400}{ }_{\text {to\& } 5 \mathrm{~min}}(4: 1)\right)$. This effect was ascribed to the scattering of the large amount of lower-density coacervate particles (with low-NPs payload) that formed because of the high excess of polymer used.[44] This is particularly evident for the (-)PSNPs suspension treated with 100:1 polymers:NPs mass ratio, whose $\% \mathrm{~T}^{400}$ to value $(22.7 \%)$ was even lower (i.e. more opaque) than that of the un- treated mixture $\left(\% \mathrm{~T}^{400}{ }_{\text {to }}\right.$ $=66.5 \%)$, indicating that immediately after stopping the stirring, the formed coacervates contribute to an increase of opacity of the suspension. 
Table 1

$\% \mathrm{~T}$ at $400 \mathrm{~nm}$ of the (-)PS-NPs suspensions $(0.0250 \mathrm{mg} / \mathrm{ml})$ after the coacervation-induced entrapment: 0 min $\left(\% \mathrm{~T}^{400} \mathrm{to}\right), 5 \mathrm{~min}\left(\% \mathrm{~T}^{400}{ }_{5 \mathrm{~min}}\right)$ and $2 \mathrm{~h}\left(\% \mathrm{~T}{ }^{400} 2 \mathrm{~h}\right)$ after stopping the stirring. $\% \mathrm{~T}{ }^{400}{ }_{24 \mathrm{~h}}$ is the $\% \mathrm{~T}$ (000 of the supernatant solutions of the treated suspensions collected after $24 \mathrm{~h}$ of standing.

\begin{tabular}{lllll}
\hline Samples & $\mathbf{\% T}^{\mathbf{4 0 0}} \mathbf{t}_{\mathbf{0}}$ & $\mathbf{\%}^{\mathbf{4 0 0}}{ }_{\mathbf{5} \text { min }}$ & $\mathbf{\%}^{\mathbf{4 0 0}} \mathbf{2 h}_{\mathbf{h}}$ & $\mathbf{\% T}^{\mathbf{4 0 0}} \mathbf{2 4 h}$ \\
\hline Untreated (-)PS-NPs & 66.5 & 66.5 & 66.5 & \\
100:1 & 22.7 & 79.4 & 92.7 & 98.8 \\
$40: 1$ & 73.4 & 93.2 & 98.6 & 99.4 \\
$4: 1$ & 96.31 & 97.7 & 97.8 & 99.1 \\
\hline
\end{tabular}

On the other hand, $2 \mathrm{~h}$ after stopping the stirring, the 3 treated sus- pensions showed similar and high transmittance values $\left(\% \mathrm{~T}^{400}{ }_{2 h}(100: 1) \cong \% \mathrm{~T}^{400}{ }_{2 h}(100: 1) \cong \% \mathrm{~T}^{400}{ }_{2 h}(4: 1)\right.$ $=92.7-98.6 \%)$, which are also very close to those of the highly transparent supernatant fractions separated after decanting the respective suspensions for $24 \mathrm{~h}$ without stir- ring $\left(\% \mathrm{~T}^{400}{ }_{24 \mathrm{~h}}=98.8-99.4 \%\right.$, Fig. 1d-e and S5, Table 1).[45] These results suggest that $2 \mathrm{~h}$ could be considered enough time to reach quantitative encapsulation and sedimentation of the suspended (-)PS-NPs. Noticeably, the lowest-concentrated G-GA solution (i.e. lowest biopolymer excess) induced the fastest clearing, due to the higher density of the formed microstructures, besides yielding the minimum sediment but equally clear supernatant fractions.

The quantitative removal of the (-)PS-NPs from the supernatants was also confirmed by DLS measurements, in which (-)PS-NPs could not be further detected (Figure S7), and SEM experiments (Figure S8) that only exhibited crystalline structures (possibly deriving from the salts present in the water) and a few large amorphous particles formed from the excess of polymer (no trace of NPs).

As reference, untreated samples and (-)PS-NPs suspensions subjected to the coacervation conditions ( $\mathrm{pH}$ and temperature) previously de-scribed, but in the absence of the trapping biopolymers, neither precipitate (\% $\mathrm{T}_{4002 \mathrm{~h}}$ remains constant, Figures $\mathrm{S9}$ ) nor changed the NPs morphology (Figures S10), confirming that the coacervates obtained from the G-GA polymers caused the encapsulation and sedimentation of the nanoparticles.

\subsection{Encapsulation and sedimentation of other families of NPs}

After the successful encapsulation and sedimentation of polymeric (-)PS-NPs in the G-GA microcapsules through complex coacervation, we aimed to test the methodology with a broader range of nanomaterials suspended in aqueous media and presenting different challenges related to their size and chemical composition (see Supporting Information for details of their synthesis). More specifically the complex coacervation was applied to:

a) a colloidal suspension of silicon oxide nanoparticles (SiOx-NPs, 0.0227 $\mathrm{mg} / \mathrm{ml}$ )[46] of average diameter of $\sim 750 \mathrm{~nm}$ as determined by SEM and DLS analyses (Figures S11). The surface of the nanoparticles is characterized by intrinsic negative charges ( $z$-potential $=-25.5 \mathrm{mV}$, Table S1). Moreover, the presence of surface hydroxyl groups may challenge the coacervation process 
through competitive electrostatic and hydrogen-bonding interactions with gelatin and/or gum Arabic.

b) b) A highly stable colloidal suspension of gold nanoparticles (Au-NPs, 0.0159 $\mathrm{mg} / \mathrm{ml}$ )[47] potentially difficult to trap and sediment due to their very small size ( $18 \mathrm{~nm}$, see TEM in Figure S12).

All above suspensions presented high colloidal stability with no precipitation for more than a week. DLS and z-potential analyses of the SiOx-NPs suspension showed no significant changes of the average size distribution and surface charge over a week without stirring (Figures S11, Table S2). The red Au-NPs suspension (Fig. 2a), with UVVis ex- tinction band at $\lambda \max =525 \mathrm{~nm}$ (Fig. 2b) was also highly stable, as demonstrated by the width and $\lambda$ max position of the band, strictly related to the Au-NPs size and aggregation state [48-49], which did not vary significantly even after months of storage (Figure S13).

Complex coacervation experiments were done using a fixed concentration of Au-NPs and SiOx-NPs NPs, while varying the polymer concentrations to ensure three different polymers:NP mass ratio (100:1, 40:1 and 4:1), as previously described. In all cases solid material concentrated at the bottom of the container upon standing, once the com- plex coacervation process was terminated (Fig. 2a and S14). Given the color of the Au-NPs suspension (due to the plasmonic extinction band at $\lambda \max =525 \mathrm{~nm}$ of the Au-NPs) the sedimentation of the encapsulated NPs could be easily corroborated also by naked-eye observation of the increase of settled purple solid and the concomitant loss of this color from the mother liquors (Fig. 2a). For both systems the amount of settled material decreased significantly upon reduction of the G-GA polymer excess, despite the suspension supernatants appeared highly clear in all cases (Fig. 2a and S14), confirming that the 4:1 mass ratio is satisfactory to induce NPs encapsulation and sedimentation.

OM and SEM analyses of the sediments revealed the formation of large spherical microcapsules (50-100 $\mu \mathrm{m})$ containing the nanomaterials when higher biopolymer ratios are used (i.e. 100:1 or 40:1 mass ratios, Fig. 2c-d and S15-S18). On the contrary, irregular-shaped microstructures at the lowest excess $(4: 1)$ were observed, in agreement with the results obtained from the encapsulation of (-)PS-NPs.

The encapsulated SiOx-NPs could be directly identified from the SEM images of the multinuclear SiOx-NPs@G-GA capsules obtained from 4:1 polymers:NPs mass ratio (Figures S18c), in which a thin layer of G-GA polymers is surrounding the encapsulated nanoparticles. On the other hand, the presence of Au-NPs in the Au-NPs@G-GA capsules was con- firmed by their purple color displayed by the OM images, which darkened as the polymers:NPs mass ratio decreases (Fig. 2d, S15), suggest- ing an increasing payload. In both cases, for the 4:1 polymers:NPs mass ratio, Au or Si could be detected by energy-dispersive X-ray spectroscopy (EDX) (Figures S19 and S20). Finally, the efficient encapsulation of SiOx-NPs was quantified by thermogravimetric analysis (TGA), from which payloads of $1.30,5.40$ and $14.9 \%$ and encapsulation efficiencies (EE) of $87.1,90.7,88.0 \%$ were determined for the 100:1, 40:1, 4:1 polymers:NPs mass ratios, respectively (Figure $\mathbf{S 2 1}$ ).

Time-dependent transmittance studies of the SiOx-NPs and Au-NPs colloidal suspensions, once the stirring was stopped after the coacervation/gelation process, revealed the clearance suffered of a similar G-GA polymers excess dependency, as for 
the (-)PS-NPs suspensions (Fig. 2e, S22-23, Table 2). In fact, very high transmittance values for the SiOx-NPs $\left(\% \mathrm{~T}^{400}{ }_{\text {to }}=88.6 \%, \% \mathrm{~T} 4005 \mathrm{~min}=96.6 \%\right)$ and Au-NPs $\left(\% \mathrm{~T}^{400}{ }_{\text {to }}=\right.$ $91.4 \%, \% \mathrm{~T} 4005 \mathrm{~min}=97.9 \%)$ suspensions were immediately achieved when the lowest excess (4:1 mass ratio) of G-GA polymers was used $\left(\% \mathrm{~T}^{400}\right.$ to\&5min $(100: 1)<\% \mathrm{~T}^{400}$ to\&5min $(40: 1)<\% \mathrm{~T}^{400}$ t0\&5min $\left.(4: 1)\right)$, confirming to be the fastest clearing condition. This was ascribed to an effective formation of high-density NPs-containing microstructures (which settle quickly) while minimizing the presence of floating of remaining NPs and/or less dense, low-payload coacervates, which are mainly produced in the presence of higher polymer excesses. Nevertheless, all suspensions were cleared after $2 \mathrm{~h}$ of standing ( $\% \mathrm{~T}_{4002 \mathrm{~h} \& 24 \mathrm{~h}}>90 \%$, Fig. 2e-f and S22-23, Table 2). The quantitative sedimentation of the Au-NPs was also corroborated by the complete disappearance of the UV-Vis extinction band from the respective supernatants (Fig. 2b), while the absence of the NPs from SiOx-NPs suspensions was confirmed by SEM of the supernatant fractions (Figures S24), which only showed the presence of unstructured amorphous material (possibly deriving from the residual G/GA polymers), or crystalline solids (related to salts present in the water).

a)

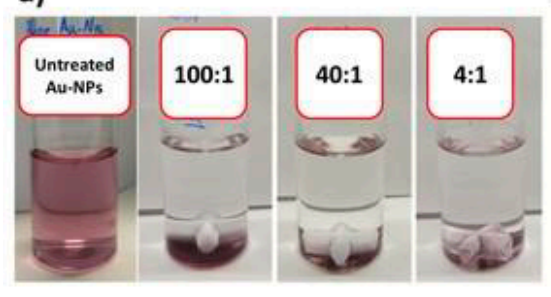

b)

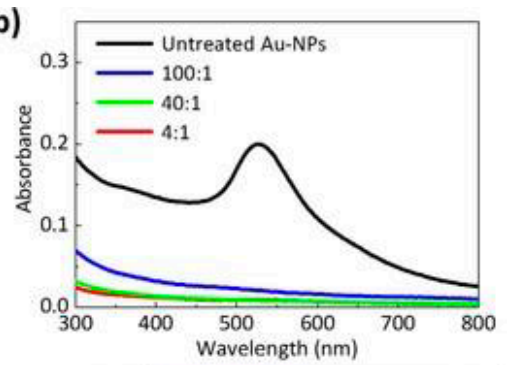

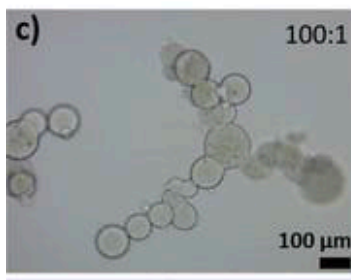

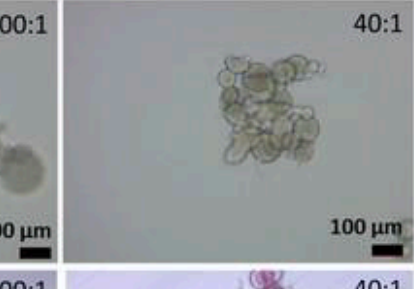

$4: 1$
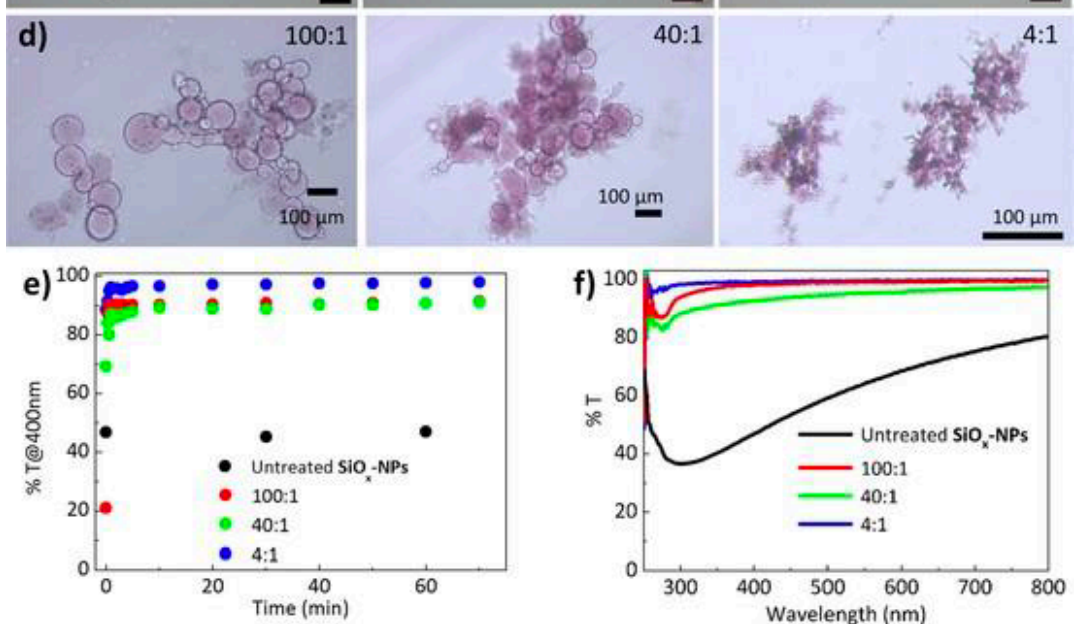

Fig. 2. a) photographs of Au-NPs suspensions $(0.0159 \mathrm{mg} / \mathrm{mL})$ before and after treatment with different polymers:NPs mass ratios (100:1, 40:1, 4:1); b) extinction spectra of Au-NPs suspensions before and after treatment with different polymers:NPs mass ratios; OM images of c) SiOx-NPs@G-GA and d) Au-NPs@G-GA microcapsules obtained upon treatment with different polymers:NPs mass ratios; e) time-dependent \%T400 of the SiOx-NPs suspensions (0.0227 $\mathrm{mg} / \mathrm{ml}$ ), untreated and after treatment with different polymers:NPs mass ratios, while standing; $f$ ) transmittance spectra of the untreated SiOx-NPs suspension $(0.0227 \mathrm{mg} / \mathrm{ml})$ and of the supernatant solutions collected $24 \mathrm{~h}$ after treatment with different polymers:NPs mass ratios, while standing. 
Table 2

\%T at $400 \mathrm{~nm}$ of the Au-NPs $(0.0159 \mathrm{mg} / \mathrm{mL})$ and SiOx-NPs $(0.0227 \mathrm{mg} / \mathrm{ml})$ suspensions after the coacervationinduced entrapment: $0 \mathrm{~min}\left(\% \mathrm{~T}{ }^{400} \mathrm{to}\right), 5 \mathrm{~min}\left(\% \mathrm{~T}^{400}{ }_{5 \mathrm{~min}}\right)$ and $2 \mathrm{~h}\left(\% \mathrm{~T}{ }^{400}{ }_{2 \mathrm{~h}}\right)$ after stopping the stirring. $\% \mathrm{~T}^{400}{ }_{24 \mathrm{~h}}$ is the $\%{ }^{400}$ of the supernatant solutions of the treated suspensions collected after $24 \mathrm{~h}$ of standing.

\begin{tabular}{|c|c|c|c|c|c|}
\hline Samples & & $\% \mathrm{~T}^{400}$ & $\% \mathrm{~T}^{400}{ }_{5 \min }$ & $\% T^{4 h}$ & $\% T^{400} 24 \mathrm{~h}$ \\
\hline Au-NPs & $\begin{array}{l}\text { Untreated } \\
\text { Au-NPs }\end{array}$ & 72.8 & 72.8 & 72.8 & \\
\hline \multirow{7}{*}{ SiOx-NPs } & 100: 1 & 41.6 & 88.3 & 96.2 & \\
\hline & $40: 1$ & 52.0 & 92.6 & 97.4 & \\
\hline & $4: 1$ & 91.4 & 97.9 & 99.3 & \\
\hline & $\begin{array}{l}\text { Untreated } \\
\text { SiOx-NPs }\end{array}$ & 46.8 & 46.8 & 46.8 & \\
\hline & 100:1 & 21.0 & 90.3 & 92.6 & 98.2 \\
\hline & 40:1 & 69.3 & 88.1 & 92.9 & 92.6 \\
\hline & $4: 1$ & 88.6 & 96.6 & 98.2 & 99.0 \\
\hline
\end{tabular}

\subsection{Effect of nanoparticles concentration}

After showing the feasibility of complex coacervation to encapsulate and sediment nanoparticles, we aimed to investigate its effectiveness at different NPs concentrations differing up to two orders of magnitude.

For this, three stable suspensions (c1 $=0.100 \mathrm{mg} / \mathrm{ml}, \mathrm{c} 2=0.249 \mathrm{mg} / \mathrm{ml}, \mathrm{c3}=2.38 \mathrm{mg} / \mathrm{ml}$ ) of model DR13-containing (-)PS-NPs were treated this time with a fixed amount of the 1:1 G-GA mixture (200 mg, $\sim 10 \mathrm{mg} / \mathrm{mL}$ ). In this way we obtain the same mass excesses of poly-mers, respect to the NPs, as those described in the previous section (100:1, 40:1 and $4: 1$, respectively), feasible for comparison purposes.

Once complex coacervation was completed and the stirring was stopped, a red solid material concentrated at the bottom of the containers in all cases (Fig. 3a, Video S1). On the other hand the supernatant fractions cleared up (\%T40024h $>95 \%$, Table S3, Fig. $3 \mathrm{~b})$, upon standing the mixtures for $24 \mathrm{~h}$ without stirring, indicating the successful encapsulation and sedimentation of the (-)PS-NPs. The absence of (-)PS-NPs or (-)PSNPs@G-GA capsules in the supernatant fractions was also corroborated by SEM and DLS measurements, which did not detect nanostructured material (Figures S25-S26). 

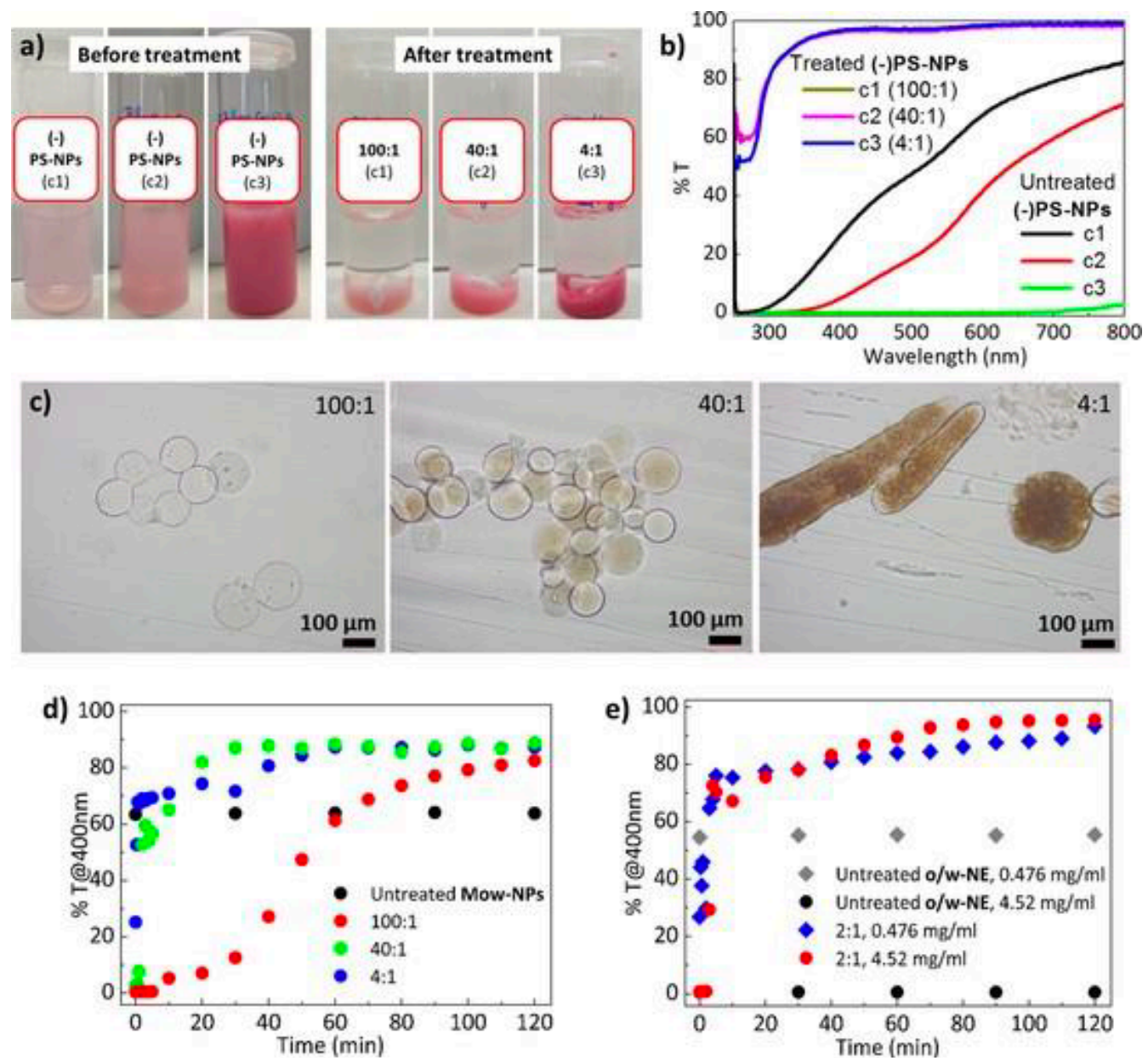

Fig. 3. a) photographs of (-)PS-NPs suspensions (NPs concentration: $c 1=0.100, c 2=0.249$ and $\mathrm{c} 3=2.38 \mathrm{mg} / \mathrm{ml}$ ) before and after coacervation-induced encapsulation using a con- stant amount of polymers ( $200 \mathrm{mg} \sim 10 \mathrm{mg} / \mathrm{ml})$, and polymers:NPs mass ratio of 100:1, 40:1 and 4:1;b) transmittance spectra of $(-)$ PS-NPs suspensions (c1 $=0.100, \mathrm{c} 2=$ 0.249 and $\mathrm{c} 3=2.38 \mathrm{mg} / \mathrm{ml}$ ) untreated and of the supernatant solutions collected $24 \mathrm{~h}$ after treatment with constant amount of polymers $(200 \mathrm{mg}, 10 \mathrm{mg} / \mathrm{ml})$, while standing; c) OM images of the corresponding (-)PS-NPs@G-GA capsules after the (-)PS-NPs encapsulation with polymers:NPs mass ratios of 100:1, 40:1 and 4:1; time-dependent \%T400 of $d)$ Mow-NPs $(0.250 \mathrm{mg} / \mathrm{ml})$ and $e)$ o/w-NE $(0.476$ and $4.52 \mathrm{mg} / \mathrm{ml})$ suspensions, untreated and after treatment with different polymers:NPs mass ratios, while standing.

OM (Fig. 3c and S27) and SEM (Figure S28) showed that the sediment was constituted by microcapsules, whose shape (spherical, of 50-100 $\mu \mathrm{m}$ size, or irregular) was, again, related to the biopolymer excess (i.e. biopolymers:NPs mass ratio), despite the higher suspended (-)PS-NPs concentration. The increase of the red color intensity of the OM images of the (-)PS-NPs@G-GA capsules and of the sediment, suggests higher payloads for the microcapsules obtained from the more concentrated NPs suspensions. This was quantitatively confirmed by $1 \mathrm{H}$ NMR measurements (Figures S29-S30) of the swollen/broken capsules obtained from the most concentrated (-)PS-NPs suspensions (i.e. 2.38 and $0.249 \mathrm{mg} / \mathrm{ml}$ ), which provided PS payloads of 18.2 and $2.40 \%$, respectively, matching with the theoretical values (20 and 2.5\%). $1 \mathrm{H}$ NMR analysis also revealed high encapsulation efficiencies ( $E E=77.7$ and $85.0 \%$, respectively), indicating, once more, the capability of the complex coacervation to encapsulate suspended NPs, even at higher concentrations. The PS in the microcapsules obtained from the lowest concentrated (- 
JPS-NPs suspension $(0.100 \mathrm{mg} / \mathrm{mL})$ could not be detected with this technique due to its sensitivity limitation.

Time-dependent \%T measurements showed the treated suspensions (after stopping the stirring) reached $\% \mathrm{~T} 400 \sim 90 \%$ in a longer time period (>60 min) than for the less concentrated NPs suspensions $(0.025 \mathrm{mg} / \mathrm{ml})$, also when the same biopolymers excess is used (i.e. 4:1, Figure S31e). This longer clearing time was ascribed to the presence of larger quantity of light scattering suspended material, deriving from

both the higher (-)PS-NPs amount to be encapsulated (the initial suspensions were much more opaque) and the higher biopolymer concentration employed, which form more amount of floating coacervates [50].

The effectiveness of the process on more concentrated nanomaterial suspensions was also corroborated on a commercial aqueous dispersion ( $120 \mathrm{~nm}$ as determined by DLS and SEM, Figure S32, Table S1) of copolymer NPs based on (meth)acrylic acid esters (Mowilith ${ }^{\circledR}$ LDM 7510, $0.250 \mathrm{mg} / \mathrm{mL}$, Mow-NPs) used for painting applications and specifically designed to have long term colloidal stability for storage (Figure S32b, Table S2).[51] Treatment with different excesses of biopolymers (keeping constant the nanoparticles concentration) yielded in all cases sedimented microcapsules (Figures S33-S35) and good clearance of the supernatants (Fig. 3d, S33 and S36, Table S4). Most of the turbidity of the suspensions was lost within two hours of standing, after the coacervation process (\%T4002 $\mathrm{h}=87.3-88.9 \%, \% \mathrm{~T} 40024 \mathrm{~h}=85.8-98.4 \%)$. Faster clearing of the aqueous suspensions (\%T4005min $(4: 1)=69.4 \%$, \%T4005 $\min (40: 1)=56.7 \%$, $\% \mathrm{~T} 4005 \mathrm{~min}(100: 1)=0.47 \%)$ and less sedimented material was accomplished with lower excess of biopolymers, as already observed for the treated (-)PS-NPs, Au-NPs and SiOxNPs suspensions.

\subsection{Effect of surface charge}

Given the relevant role of electrostatic interactions in the complex coacervation process, we aimed to investigate the effects of the variation of nanomaterial surface charge on their encapsulation and posterior sedimentation. For this study, we selected the DR13-containing polystyrene nanoparticles ( $260 \mathrm{~nm}$, Figure S37, Table S1), which could be obtained with positive charge (+36.2 mV, Tables S1 and S2) by simply substituting the SDS surfactant with cetyltrimethylammonium bromide (CTAB) during their synthesis ((+)PS-NPs, see Supporting Information).

Complex coacervation with 4:1 polymers:NPs mass ratio conditions (which produced the fastest encapsulation/sedimentation in the previous NPs suspensions), was carried out on two different stable (+)PS-NPs suspensions $(0.0250 \mathrm{mg} / \mathrm{ml}$ and $2.38 \mathrm{mg} / \mathrm{ml})$. Treatment with the biopolymers yielded very clear supernatant solutions $(\% \mathrm{~T} 40024 \mathrm{~h}=$ 96.7 and $88.3 \%$, \% $>85 \%$ in all visible range, Figure S38) and a very quick precipitation of (+)PS-NPs@G-GA at the bottom of the vials (Figure S39), consisting of shapeless microstructures whose coloration increased with the concentration of the initial suspension (Figures S40-S41). The (+)PS-NPs@G-GA capsules payload (22.1\%, matching with the theoretical value) and EE (96.4\%), determined by $1 \mathrm{H}$ NMR (for the most concentrated system, Figure S42) were very high, as in the case of the (-)PS-NPs@G-GA microcapsules obtained from the treatment of the (-)PS-NPs suspension of same concentration $(2.38 \mathrm{mg} / \mathrm{ml})$. Sedimentation profiles also resembled those of the treated (-)PS-NPs suspensions of same respective concentrations (i.e. $0.0250 \mathrm{mg} / \mathrm{mL}$ and 2.38 
$\mathrm{mg} / \mathrm{mL}$ ), with shorter clearing times observed for the less concentrated NPs suspension (\%T4005 $\mathrm{min}=88.9$ and $9.40, \% \mathrm{~T} 4002 \mathrm{~h}=94.7$ and $77.9 \%$, respectively, Figures $38 \mathrm{c}$, Table S5). Noticeably, these re- sults suggest that the NPs surface charge does not affect significantly the complex coacervate formation, the encapsulation and the sedimentation processes. This is an important advantage compared to other coagulating/flocculating methods (e.g. neutralization), whose efficiency is strictly related to the electrostatic interactions between the nanoparticles and polymers.

\subsection{Encapsulation and sedimentation of oil nanodroplets}

To demonstrate the universality of our approach, we also challenged the encapsulation and sedimentation of liquid oil nanodroplets dispersed in water. Such oilin-water nanoemulsions (o/w-NE) are often employed in drug delivery, cosmetic and food products, and the lower density of the oil droplets compared to water makes them quite difficult to settle [52-53]. For this study two differently concentrated o/w nanoemulsions $\left(0.476\right.$ and $4.52 \mathrm{mg} / \mathrm{ml}$ ) were prepared by emulsifying Miglyol ${ }^{\circledR} 812$ oil (a triglyceride of capric/caprylic acid) in a surfactant-containing water solution, through ultrasonication (see experimental details in Supporting Information) [52-54]. The resulting nanodroplets ( $150 \mathrm{~nm}$ ) showed very high colloidal stability upon several days (see DLS in Figure S43, Table S2). In spite so, the nanodroplets were quickly encapsulated and sedimented (Figures S44-S46), yielding clear supernatants within $2 \mathrm{~h}$ of standing (\%T4002 $\mathrm{h}=93.2 \%$ and $95.5 \%$, respectively) for both o/w-NEs (Fig. 3e and S47, Table S6). Noticeably, the process resulted effective, despite the polymers:NPs mass ratio was decreased to 2:1.1H NMR analysis of swollen and broken o/w-NE@GGA capsules obtained from the two treated nanoemulsions showed Miglyol ${ }^{\circledR} 812$ payloads matching with the theoretical values (33.0 and 32.4\%, Figure S48) and EE as high as 98.0 and $88.8 \%$ respectively.

\subsection{Complex coacervation for micro/nanomaterial separation from aqueous residues}

The efficient encapsulation of the different types of nanomaterials and their quick sedimentation incentivized us to explore complex coacervation for the separation/removal of aqueous solid micro/nanocontaminants, as potential alternative method to the normally used inorganic coagulant/flocculant agents, which present toxicity issues, or cost/ time-consuming synthetic modification of biopolymers. To carry on this study, we first applied the method to a model suspension prepared from a mixture of the previous nanoparticles (Mixed-NPs), simulating a model aqueous residue, and successively to two real water-based wastes (Lab1-WE and Lab2-WE) generated in our laboratories along daily re- search activity.

The Mixed-NPs sample was obtained by mixing (-)PS-NPs, SiOx-NPs, Au-NPs and o/w-NE with a mass ratio of 1.4:1.4:1.0:2.7 and overall concentration of $0.1 \mathrm{mg} / \mathrm{ml}$. Despite the simultaneous presence of NPs differing in dimensions $(10-700 \mathrm{~nm})$, material (metallic, polymeric, oxide) and state (solid or liquid), treatment of the sus- pension with the mixture of polymers (polymers:NPs mass ratio $4: 1$ ) yielded settled capsules and quite clear supernatants $(\% \mathrm{~T} 4002 \mathrm{~h}=82.2, \% \mathrm{~T} 40024 \mathrm{~h}=98.7 \%$, Fig. 4a and S49-S51, Table S7), with no detectable absorption band at $525 \mathrm{~nm}$ (related to the Au-NPs band and encapsulated DR13 in (-)PS-NPs). 
Lab1-WE and Lab2-WE mixtures contained random composition of emulsified oils and volatile organic solvents (minor amount given the water-based nature of the waste), micro/nanoparticles of different types, size and surface charge, dissolved dyes, polymers, reactants, salts, etc. Both mixtures were relatively stable (most of the material did not sediment for several days), milky due to the presence of a high concentration of suspended and/or partially aggregated micro/nanoparticles (Figure S52) and colored (because of the presence of suspended pigments or dissolved dyes) suspensions (Fig. 4b and S53). Known aliquots of these wastes were first freeze-dried to estimate the total amount of solids and non-volatile liquids (TSLs) contained in the mixtures (240.6 and $315.3 \mathrm{mg}$ ), through gravimetric measurements. Based on these values, suspensions of the two waste effluents $(1.59 \mathrm{mg} / \mathrm{ml})$ were treated with the G-GA polymer mixture. Despite the two wastes were prepared with the same concentration $(1.59 \mathrm{mg} / \mathrm{ml})$, the higher transparency of the untreated Lab2-WE was mainly ascribed to a much higher amount of water soluble solids in the composition, which do not contribute to the light scattering (Table S7).

In water remediation, the coagulant/flocculants amount is usually optimized to minimize the settled material and cost. For this reason, much lower amounts of G-GA polymers (as low as $160 \mathrm{mg} / \mathrm{l}$ ) than what used in the previous experiments, were also attempted (Lab1-WE, 0.1:1 and 1:1, while in Lab2-WE, 0.4:1 and 4:1 polymers:TSLs mass ratios) to encapsulate and sediment the contaminants of the water wastes.

Complex coacervation produced in all cases Lab1-WE@G-GA and Lab2-WE@G-GA capsules (see SEM and OM in Figures S54-S55) that entrapped and settled at the bottom of the vials with the dispersed ma- terials (Fig. $4 \mathrm{~b}$ and S53). Sedimentation rate of the treated wastes was dependent on the used polymers:TSLs mass ratios conditions (Fig. 4c, Figure S53b, Table S7), especially for the Lab1-WE, in which an ex- pected slower settling was observed when a defect amount of biopoly- mers was employed (\%T4002h $(0.1: 1)=11.6 \%, \% \mathrm{~T} 4002 \mathrm{~h}(1: 1)=78.9$, Table S7). Nevertheless, the overall encapsulation [55] of the dis- persed material in Lab1-WE $(\mathrm{EE}(0.1: 1)=74.4 \%$, EE $(1: 1)=71.1 \%)$ and Lab2-WE (EE $(0.4: 1)=50.0 \%, \mathrm{EE}(4: 1)=56.6 \%)$ and their sed- imentation after $24 \mathrm{~h}$ (Lab1-WE, \%T40024h = 82.8-84.9; Lab2-WE, \%T40024h = 87.3, Table S7) were equally efficient (the lower EE deter- mined for the Lab2-WE was ascribed to a higher content of solid wa-ter-soluble materials in the initial composition, which was not trapped through this method), even with 10 times less biopolymers amount (Fig. 4b). 

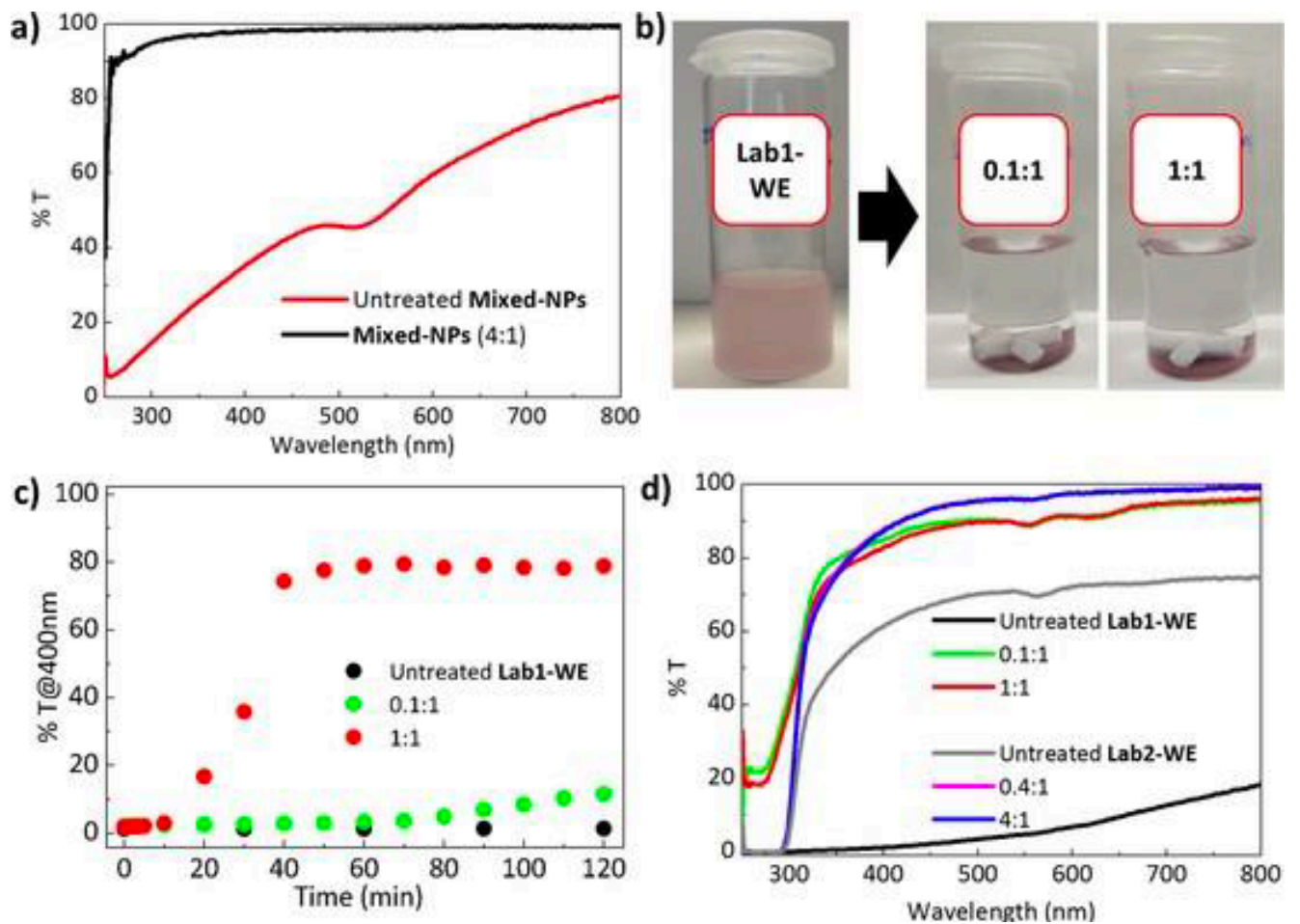

Fig. 4. a) transmittance spectra of the Mixed-NPs $(0.1 \mathrm{mg} / \mathrm{ml})$ suspension before treatment and of the supernatant solution of the treated suspension kept standing $24 \mathrm{~h}$ without stirring; b) photographs of Lab1-WE $(1.59 \mathrm{mg} / \mathrm{ml})$ before and after treatment with 0.1:1 and 1:1 polymers:TSLs mass ratios; $c$ ) time-dependent \%T400 of the untreated and treated Lab1-WE, while standing; d) transmittance spectra of the Lab1-WE and Lab2-WE before treatment (1.59 $\mathrm{mg} / \mathrm{ml}$ ) and of the supernatant solutions of the treated suspensions kept standing $24 \mathrm{~h}$ without stirring.

\section{Conclusions}

In summary, we have demonstrated that the in-situ formation of a polymeric network from oppositely charged and commercially avail- able macromolecules (gelatin and gum Arabic) is a straightforward and non-specific strategy for the efficient entrapment and sedimentation of nanomaterials dispersed in aqueous media, without further chemical modifications. As hypothesized, the coacervates physically entrap sus- pended nanomaterials with high efficiency (approaching the theoretical values) and quickly sediment (within two hours in most of the cases) after gelation, resulting in the clearance (\%T 90\%) of highly stable colloidal suspensions. Higher payloads, faster clearings and reduced sediments, but similarly transparent supernatants, were obtained with lower biopolymers excess. The methodology also revealed to be a potential alternative to de-emulsify stable oil-in-water nanoemulsions [56-57] and preliminary experiments suggest it can be effective for the concentration and removal of a random mixture of micro/nanomaterials from real aqueous residues.

The coacervation, successfully applied to nanomaterials that differed for their chemical nature (organic/inorganic, solid/liquid), size (10-700 nm) and surface charge, revealed more general than other particles surface charge-dependent mechanisms (e.g. neutralization or patch), normally involved in standard flocculation/coagulation strategies with fixed charge polymers or inorganic salts [22-25,28-35]. Moreover, although the used polymers concentrations are higher than in some of the standard flocculation/coagulation methods $(\sim 0.1-400 \mathrm{mg} / \mathrm{mL})$ [28], this strategy overcomes i) health and environmental issues [22] and the low efficiency towards very fine particles 
of multivalent inorganic salts (e.g. Al, Fe, etc.), [26-28] or ii) the need of cost/ time consuming syntheses/modification of organic polyelectrolytes (e.g. polyacrylamides, grafted chitosan, starch, etc.), which also lack of biodegradability [25,28,32]. Ongoing studies are showing the reversible electrostatic interaction between the polyelectrolytes could be exploited to revert the process and permit the release (and recovery) of concentrated high-value nanomaterials and/or biopolymers recycling, usually not possible with fixed charge polymers [26-27]. Future research should focus on adapting the process to macromolecules that al- low operating at higher $\mathrm{pHs}$, room temperature and lower polymer excesses, to minimize costs.

\section{Acknowledgements}

This work was supported by grants from the Spanish Research Agency funds (AEI, grant no. RTI2018-098027-B-C21), by the Euro- pean Regional Development Fund (ERDF) and BIST (BIST Ignite Project). The ICN2 is funded by the CERCA programme/Generalitat de Catalunya. The ICN2 is supported by the Severo Ochoa Centres of Excellent programme, funded by the Spanish Research Agency (AEI, grant no. SEV-2017-0706). We also acknowledge J. R. Otaegui, A. Julià López and B. García for the help in the synthesis of some of the used nanomaterials.

\section{References}

Blackman, L.D., Gunatillake, P.A., Cass, P., Locock, K.E.S., 2019. Chem. Soc. Rev. 48, 757-770.

Timilsena, Y.P., Akanbi, T., Khalid, N., Adhikari, B., Barrow, C.J., 2019. Int. J. Biol. Macromol. 121, 1276-1286.

Sing, C.E., Perry, S.L., 2020. Soft Matter 16, 2885-2914.

van der Gucht, J., Spruijt, E., Lemmers, M., Cohen Stuart, M.A., 2011. J. Colloid Interface Sci. 361, 407-422.

de Kruif, C.G., Weinbrecka, F., de Vries, R., 2004. Curr. Opin. Colloid Interface Sci. 9, 340-349.

Kizilay, E., Kayitmazer, A.B., Dubin, P.L., 2011. Adv. Colloid Interface Sci. 167, 24-37. Xu, Y., Mazzawi, M., Chen, K., Sun, L., Dubin, P.L., 2011. Biomacromolecules 12 (5), 1512-1522.

Eghbal, N., Saeid Yarmand, M., Mousavi, M., Degraeve, P., Oulahal, N., Gharsallaoui, A., 2016. Carbohydr. Polym. 151, 947-956.

Lalevée, G., David, L., Montembault, A., Blanchard, K., Meadows, J., Malaise, S., Crépet, A., Grillo, I., Morfin, I., Delair, T., Sudre, G., 2017. Soft Matter 13, 6594.

Tsogas, G.Z., Giokas, D.L., Vlessidis, A.G., 2010. Talanta 80, 2049-2056.

Dompé, M., Cedano-Serrano, F.J., Heckert, O., van den Heuvel, N., van der Gucht, J., Tran, Y., Hourdet, D., Creton, C., Kamperman, M., 2019. Adv. Mater. 31, 1808179.

Schmitt, C., Turgeon, S.L., 2011. Adv. Colloid Interface Sci. 167, 63-70.

Timilsena, Y.P., Wang, B., Adhikari, R., Adhikari, B., 2017. Food Hydrocolloids 69, 369-381.

Ghayempour, S., Montazer, M., 2016. J. Microencapsulation 33, 497-510. Saravanana, M., 2010. K. Panduranga Rao. Carbohydr. Polym. 80, 808-816. Hawladera, M.N.A., Uddina, M.S., Khin, M.M., 2003. Appl. Energy 74, 195-202.

Dong, Z.J., Touré, A., Jia, C.S., Zhang, X.M., Xu, S.Y., 2007. J. Microencapsulation 24, 634-646.

Yeo, Y., Bellas, E., Firestone, W., Langer, R., Kohane, D.S., 2005. J. Agric. Food Chem. 53, 7518-7525.

Dong, Z., Ma, Y., Hayat, K., Jia, C., Xia, S., Zhang, X., 2011. J. Food Eng. 104, 455-460. Lemetter, C., Meeuse, F., Zuidam, N., 2009. AICHE J. 55, 1487-1496.

Pei, Y., Zheng, Y., Li, Z., Liu, J., Zheng, X., Tang, K., Kaplan, D.L., 2021. J. Colloid Interface Sci. 582, 610-618.

Teh, C.Y., Budiman, P.M., Yee Shak, K.P., Wu, T.Y., 2016. Ind. Eng. Chem. Res. 55, 4363-4389.

Zhou, D., Abdel-Fattah, A., Keller, A.A., 2012. Environ. Sci. Technol. 46, 7520-7526. Sahu, O.P., Chaudhari, P.K., 2013. J. Appl. Sci. Environ. Manage. 17, 241-257.

S. Jabin, J.K. Kapoor, Role of Polyelectrolytes in the Treatment of Water and Wastewater. In: Inamuddin, Asiri A. (Eds.), Sustainable Green Chemical Processes and their Al- lied Applications. Nanotechnology in the Life Sciences, 2020, Springer, Cham. https:// doi.org/10.1007/978-3-030-42284-4_10.

Leshuk, T., Holmes, A.B., Ranatunga, D., Chen, P.Z., Jiang, Y., Gu, F., 2018. Env. Sci. Nano 5, 509-519.

Kimura, M., Matsui, Y., Kondo, K., Ishikawa, T.B., Matsushita, T., Shirasaki, N., 2013.

Water Res. 47, 2075-2084.

Lee, C.S., Chong, M.F., Robinson, J., Binner, E., 2014. Ind. Eng. Chem. Res. 53, 18357-18369. 
Yang, R., Li, H., Huang, M., Yang, H., Li, A., 2016. Water Res. 95, 59-89. Bolto, B., Gregory, J., 2007. Water Research 41, 2301-2324.

Morrissey, K.L., He, C., Wong, M.H., Zhao, X., Chapman, T.Z., Bender, S.L., Prevatt, W.D., Stoykovich, M.P., 2015. Biotechnol. Bioeng. 112, 74-83.

Kinyua, E.M., Mwangi, I.W., Wanjau, R.N., Ngila, J.C., 2016. Environm. Sci. Pollut. Res. 23, 5214-5221.

Gupta, B.S., Ako, J.E., 2005. Eur. Food Res. Technol. 221, 746-751.

Grenda, K., Arnold, J., Gamelas, J.A.F., Cayre, O.J., Rasteiro, M.G., 2020. Sep. Purif.

Technol. 231, 115888 .

Shaikh, S.M.R., Nasser, M.S., Hussein, I., Benamor, A., Onaizi, S.A., Qiblawey, H., 2017.

Sep. Purif. Technol. 187, 137-161.

Zhao, W., Fan, Y., Wang, H., Wang, Y., 2017. Langmuir 33, 6846-6856.

Pérez-Calderón, J., Santos, M.V., Zaritzkya, N., 2018. J. Environ. Chem. Eng. 6, 6749-6760.

Valley, B., Jing, B., Ferreira, M., Zhu, Y., Appl, A.C.S., 2019. Mater. Interfaces 11, 7472-7478.

Yang, Y., Anvari, M., Pan, C.-H., Chung, D., 2012. Food Chem. 135, 555-561.

Staff, R.H., Schaeffel, D., Turshatov, A., Donadio, D., Butt, H.-J., Landfester, K., Koynov, K., Crespy, D., 2013. Small 9, 3514-3522.

López, A.J., Ruiz-Molina, D., Landfester, K., Bannwarth, M.B., Roscini, C., 2018. Adv.

Funct. Mater. 1801492.

Sarika, P.R., Pavithran, A., James, N.R., 2015. Food Hydrocolloids 49, 176-182. Lv, Y., Zhang, X., Abbas, S., Karangwa, E., 2012. J. Food Eng. 111, 225-233.

The oscillations observed in the first 1-3 spectra measured soon after stopping the stir- ring (short period of time) are due to the fluctuation of the number of large particles passing through the detection light beam during the measurements (wavelength scan- ning).

The presence of the band at $280 \mathrm{~nm}$ is due to G-GA dissolved in water, being decreased with the reduction of biopolymer used for the NPs removal.

Wang, Q., Liua, Y., Yan, H., 2007. Chem. Commun. 2339-2341.

Vodnik, V.V., Abazović, N.D., Stojanović, Z.A., Marinović-Cincović, M., Mitrić, M., Comor, M.I., 2011. J. Compos. Mater. 46, 987-995.

Choudhary, A., Singh, G., Biradar, A.M., 2014. Nanoscale 6, 7743-7756.

Zeng, S., Yong, K.-T., Roy, I., Dinh, X.-Q., Yu, X., Luan, F., 2011. Plasmonics 6, 491-506.

Immediately after stopping the stirring (t0) a decrease of transmittance was observed for the suspensions treated with larger excess of G-GA (especially for the case of 100:1 polymers:NPs ratio) respect to the untreated suspensions (i.e. \%T400t0 <\%T400 of untreated NPs). This is due to the high scattering contribution of the coacervates formed from the excess of the polymers respect to the NPs.

https://uk.ravagochemicals.com/wp-content/uploads/2017/07/CELANESE-Paints- Coatings-Brochure.pdf, last access 05/11/2020.

Singh, Y., Gopal Meher, J., Raval, K., Khan, F.A., Chaurasia, M., Jainc, N.K., Chourasia, M.K., 2017. J. Controlled Release 252, 28-49.

Gupta, A., Eral, H.B., Hatton, T.A., Doyle, P.S., 2016. Soft Matter 12, 2826-2841. Torres-Pierna, H., Ruiz-Molina, D., Roscini, C., 2020. Mater. Horiz. 7, 2749-2759.

TSLs entrapment efficiency was obtained by the weight difference between the treated TSL effluents (calculated by subtracting the initial amount of G-GA from the freeze-dried Lab1-WE@G-GA and Lab2-WE@G-GA microcapsules weight) and the freeze-dried untreated TSLs.

Barry, E., Mane, A.U., Libera, J.A., Elamb, J.W., Darling, S.B., 2017. J. Mater. Chem. A. 5, 2929-2935. Zolfaghari, R., Fakhrúl-Razi, A., Abdullah, L.C., Elnashaie, S.S.E.H., Pendashteh, A., 2016. Sep. Purif. Technol. 170, 377-407. 